\title{
Flapping Wing Devices for Mars Exploration
}

\author{
Asier Ania* \\ Royal Military College of \\ Canada \\ Asier.Ania@rmc.ca
}

\author{
Dominique Poirel \\ Royal Military College of \\ Canada \\ Poirel-d@rmc.ca \\ Steeve Montminy \\ Canadian Space Agency \\ Steeve.Montminy@space.gc.ca
}

\author{
Marie-Josée Potvin \\ Canadian Space Agency \\ Marie- \\ Josee.Potvin@space.gc.ca
}

\begin{abstract}
The use of an aerial vehicle would greatly enhance the domain of exploration on Mars. The main constraint in such a design would be the extreme Martian environment. The low-density atmosphere suggests the use of a low Reynolds number flight regime modeled after flapping wing insect flight. This flapping wing flight employs several unsteady aerodynamic mechanisms; delayed stall, wake capture, and rotational mechanisms. Two prototypes, a flapping wing and a rotary-flapping wing hybrid, have been built and will be tested in order to quantify the 'overall lift' generated and allow us to evaluate the efficacy of flapping wing flight on Mars.
\end{abstract}

\section{Introduction}

$\mathrm{C}_{\mathrm{r}}^{\mathrm{u}}$ URRENT robotic missions to Mars involve relatively large rovers covering a few hundred square meters of ground. An alternate method of exploring a greater area on Mars would be to use an aerial vehicle. Previous Mars aerial vehicle concepts include balloon designs, fixed wing aircraft, and rotary wing aircraft (helicopter). Balloon designs have limited controllability because they yield to the predominant weather patterns; they are also more costly. Fixed wing aircraft require great speeds to achieve the necessary sustaining vertical force. Rotary wing aircraft are being explored by certain groups [1] and appears to have some potential for Mars flight even though the speed of sound is lower. The objective of this study is to determine the feasibility of using flapping wing devices for flight on Mars; a proof of concept. The following paper outlines the aerodynamic theory, the current prototypes and the next steps involved in the project.

\section{Background}

One benefit of flying on Mars is that the gravity is lower than Earth's, however both values are of the same magnitude. The atmospheric density on Mars is two orders of magnitude lower than Earth's atmosphere (near the surface). It is this lower atmospheric density that provides the main design constraint for a Mars aerial vehicle.

Table 1: Properties of Mars and Earth $[1,2,3]$

\begin{tabular}{|l|l|l|}
\cline { 2 - 3 } \multicolumn{1}{c|}{} & Mars & Earth \\
\hline Mass [Kg] & $6.42 \times 10^{23}$ & $5.98 \times 10^{24}$ \\
\hline Radius [m] & $3.39 \times 10^{6}$ & $6.38 \times 10^{6}$ \\
\hline Gravity [g] & 0.38 & 1 \\
\hline
\end{tabular}

\begin{tabular}{|c|c|c|}
\hline \multicolumn{3}{|c|}{ Atmosphere (near the surface) } \\
\hline Density $\left[\mathrm{Kg} / \mathrm{m}^{3}\right]$ & 0.0142 & 1.225 \\
\hline Pressure $[\mathrm{Pa}]$ & 680 & 101350 \\
\hline Temperature $[\mathrm{K}]$ & 250 & 288 \\
\hline $\begin{array}{l}\text { Dynamic Viscosity } \\
{\left[\mathrm{Ns} / \mathrm{m}^{2}\right]}\end{array}$ & $1.2 \times 10^{-5}$ & $1.789 \times 10^{-5}$ \\
\hline $\begin{array}{l}\text { Kinematic Viscosity } \\
{\left[\mathrm{m}^{2} / \mathrm{s}\right]}\end{array}$ & $8.45 \times 10^{-4}$ & $1.46 \times 10^{-5}$ \\
\hline $\begin{array}{l}\text { Speed of Sound } \\
{[\mathrm{m} / \mathrm{s}]}\end{array}$ & 247 & 340 \\
\hline Composition [\%] & $\begin{array}{l}\mathrm{CO}_{2}[95] \\
\mathrm{N}_{2}[2.7] \\
\mathrm{Ar}[1.6] \\
\text { Other [0.7] }\end{array}$ & $\begin{array}{l}\mathrm{N}_{2}[79] \\
\mathrm{O}_{2}[20] \\
\text { Other [1] }\end{array}$ \\
\hline
\end{tabular}

* Author for Correspondence

The lower atmospheric density suggests the use of a low Reynolds number flight regime (see Background Theory). This leads to an investigation of insect flight 
that operates at a low Reynolds number in the range of 10 to 10000 [4]. Insects employ a combination of conventional and non-conventional aerodynamic mechanisms in order to create the sustaining vertical force required for flight. The 'sustaining vertical force' will be denoted here by the term 'overall lift' which should be interpreted differently from the term 'lift'. Whereas 'overall lift' defines the sustaining vertical force required for flight of the entire vehicle or insect. The term 'lift' will be reserved for discussion of the individual lift vector (perpendicular to the oncoming flow) resulting from the movement of each wing through its wing cycle. The non-conventional or unsteady aerodynamic mechanisms employed in flapping wing flight have been the topic of much research in recent years. An excellent and thorough examination of the aerodynamics of insect flight was written by C.P. Ellington $[5,6,7,8,9,10]$ and a recent concise review of the topic was written by S.P. Sane [11].

It is important to note that this project is not intended to replicate the flight of an insect. Biomimicry is not the objective, rather, the use of a design oriented approach that exploits the mechanisms insects use in order to maintain flight is the goal.

\section{Background Theory}

\section{Reynolds Number}

The 'Reynolds number' ( $\mathrm{Re}$ ) is a non-dimensional parameter that relates the inertia forces to the viscous forces in a fluid flow. It appears naturally in the nondimensional form of the governing equations of the fluid flow; the Navier-Stokes equations. The $\mathrm{Re}$ is used to characterize flight regimes for various aerial vehicles. Its nominal expression is:

$$
\operatorname{Re}=\frac{\rho U L}{\mu}
$$

$U[\mathrm{~m} / \mathrm{s}]$ and $L[\mathrm{~m}]$ are the characteristic velocity and length scales of the flow, respectively (determined by the aerial vehicle). $\rho\left[\mathrm{kg} / \mathrm{m}^{3}\right]$ and $\mu\left[\mathrm{Ns} / \mathrm{m}^{2}\right]$ are the density and dynamic viscosity of the fluid, respectively. Since the dynamic viscosity of the Martian atmosphere relative to Earth's is of the same order of magnitude and the atmospheric density is two orders of magnitude lower, the Re on Mars is lower for the same aerial vehicle. This low Re has a direct effect on the lift capability of the vehicle. As expressed by equation 2 the lift coefficient is a function of Reynolds number and other variables [12].

$C_{L}=C_{L}(\operatorname{Re}, M$, shape,$\alpha)$

$M$ is the Mach number and $\alpha$ is the angle of attack. Fixed wing aircraft operate at large Re. In order to generate the same lift coefficient by maintaining the same Re for Mars flight, the velocity and size of the vehicle need to be increased almost two orders of magnitude combined. Furthermore, an equivalent lift coefficient, $C_{L}$, does not guarantee sufficient lift since the lift force depends also directly on the fluid density as shown by equation 3 [12]:

$$
L=1 / 2 \rho U^{2} S C_{L}
$$

$S$ is the planform area of the wing. Accordingly, a higher lift coefficient is required to compensate for the lower density. Flapping wing insect flight lies within a low Re regime. Higher lift coefficients, than those based on fixed wing conventional aerodynamics, are created. Flapping wings can therefore become a suitable model for designing a Mars aerial vehicle.

\section{Conventional Aerodynamics}

Fixed wing aerodynamics is often studied with the aid of a simplified 2-D airfoil model that assumes that the flow is two-dimensional. Figure 1 shows a 2-D airfoil at a geometric angle of attack, $\alpha$, relative to the free stream velocity vector $\mathrm{U}_{\infty}$, that represents the oncoming airflow. Note that in a 3-D fixed wing, downwash created by tip vortices would slightly decrease the 'effective' angle of attack. The resultant aerodynamic force is broken up into two conventional components. The lift force is perpendicular to the $\mathrm{U}_{\infty}$ direction, and the drag force is in the same direction of $\mathrm{U}_{\infty}$ and acts to oppose the forward movement of the wing. In this example the lift force generated on the wing is the reason for the sustaining vertical force or 'overall lift' that keeps the aircraft in the air. The above example and terminology allows us to expand our framework of analysis to encompass more real and complex wing motions that involve flapping.

\section{Flapping Wings}

The lift force generated by the wings of a fixed wing aircraft creates the sustaining vertical force ('overall lift') and the engine provides the thrust. In flapping wing flight both the 'overall lift' and thrust is generated by the flapping of the wings. Figure 2 


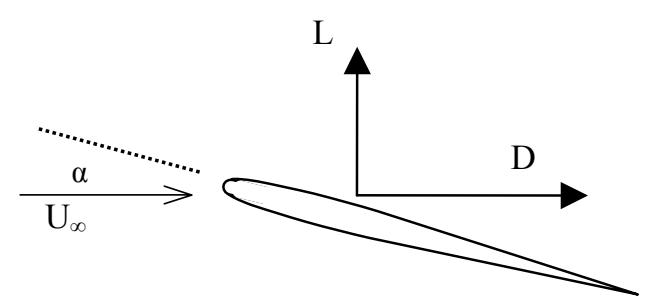

Figure 1. Aerodynamic forces on an airfoil.

depicts an insect in flight. The entire wing cycle (or wing beat) can be separated into the translation phase and stroke reversal or rotation phase. The translation phase consists of the downstroke and upstroke; the downstroke is usually defined as dorsal to ventral wing stroke. The wing reversal phase consists of the rotational movements of the wing where supination is defined as going from the downstroke to the upstroke (see Figure 2) and pronation is defined as going from the upstroke to the downstroke. The motion of the wing itself induces its own relative airflow velocity vector.

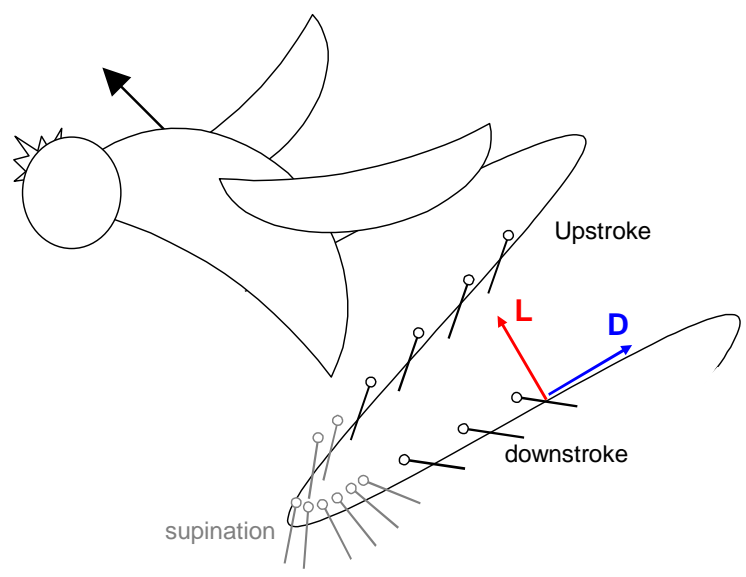

Figure 2. Insect in flight. Adapted from Ellington [4]. The circle on the end of the wing cross section (black or grey line) represents the leading edge of the wing.

As can be seen in Figure 2 on the first downstroke wing position the resultant oncoming airflow produces a lift vector that contributes to the thrust (forward movement) and 'overall lift' and the drag vector also contributes to the 'overall lift' of the insect. The amount of contribution of each force component vector (lift and drag) towards either the thrust or 'overall lift' is dependent on the movement and orientation of the wing and hence on the wing kinematics. Insect wing kinematics is very complicated and stems from an evolutionary adapted control mechanism that allows the insect to independently alter on each wing, various wing parameters at various times throughout the wing cycle that ultimately determine the time course of flight forces. The wing parameters are: stroke amplitude, flapping frequency, angle of attack, mean stroke plane, deviation from mean stroke plane, wing tip trajectory, and timing \& duration of wing reversal [11]. It is also important to point out that insect wings are flexible and not rigid, hence the passive parameter of wing camber is important, as well as any aeroelastic effects that have not been extensively studied thus far. The mean $\mathrm{Re}$ for hovering flapping flight defined by Ellington [4] is:

$\operatorname{Re}=\frac{\bar{c} \bar{U}_{t}}{v}=\frac{4 \phi n R^{2}}{v(A \Re)}$

Where $\phi$ is the stroke amplitude [rad], $n$ is the flapping frequency (cycle/s), $R$ is the length of the wing $[\mathrm{m}]$, the kinematic viscosity is $v=\mu / \rho\left[\mathrm{m}^{2} / \mathrm{s}\right]$ and the aspect ratio, $A \Re=4 \mathrm{R}^{2} / \mathrm{S}[8]$ where $\mathrm{S}=$ surface area of the pair of wings $\left[\mathrm{m}^{2}\right]$. The characteristic length is given by the mean chord, $\bar{c}=2 \mathrm{R} / A \Re[\mathrm{m}]$ and the characteristic velocity is given by the mean wingtip velocity, $\overline{\mathrm{U}}_{\mathrm{t}}=2 \phi n \mathrm{R}[\mathrm{m} / \mathrm{s}]$.

The investigation of hovering flight eliminates the forward thrust variable summed-up over one full cycle, simplifies the wing parameters and focuses on the balance between the 'overall lift' and the weight of the insect. Early work in studying the aerodynamic forces in insects focused on a quasi-steady state aerodynamic model [5]. The quasi-steady state model separates the wing path into discrete instantaneous positions while the steady state forces are calculated based on the local parameters (angle of attack, wing geometry, relative fluid velocity) at each instantaneous position along the path. This model has shown that conventional steadystate aerodynamic analysis is insufficient in accounting for the 'overall lift' produced by insects [5]. In some instances the wings can produce 2-3 times the force accounted for by conventional aerodynamic theory [4]. This leads into the investigation of alternative lift producing mechanisms of unsteady aerodynamics. Recent efforts at revising the quasi-steady state model to incorporate the wing 'rotational forces' have improved upon the accuracy of such models [13]. 


\section{Unsteady Aerodynamics}

There are various time dependent aerodynamic phenomena that account for the observed high 'overall lift' in insect flight. The main phenomena are delayed stall, wake capture and rotational mechanisms and are used by insects at various times in the wing cycle and at various stages in flight in order to produce the desired maneuverability and the necessary sustainable vertical force.

\section{Delayed Stall}

Delayed Stall occurs during the translation phase of the wing cycle (i.e. in the downstroke). The process is similar to that of dynamic stall; the high lift regime of post static stall angles of attack on fixed and rotary wings. As the wing translates through a downstroke at high angles of attack the oncoming airflow reaches the leading edge and separates from the wing and then

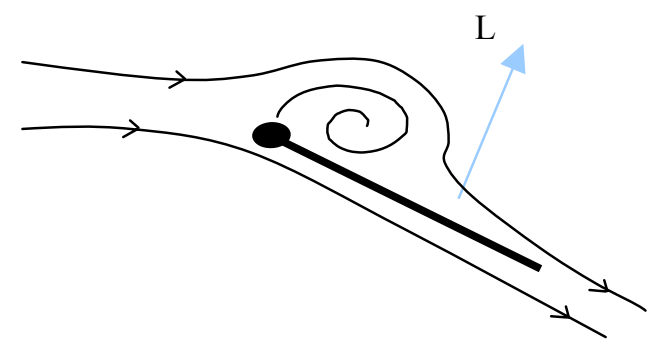

Figure 3. Leading edge vortex on a

wing.. Adapted from Dickinson, and Sane [11].

reattaches further down the wing. This is a result of a
1

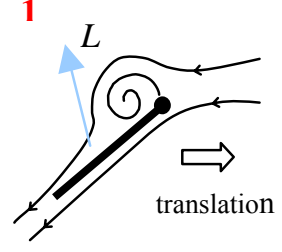

2
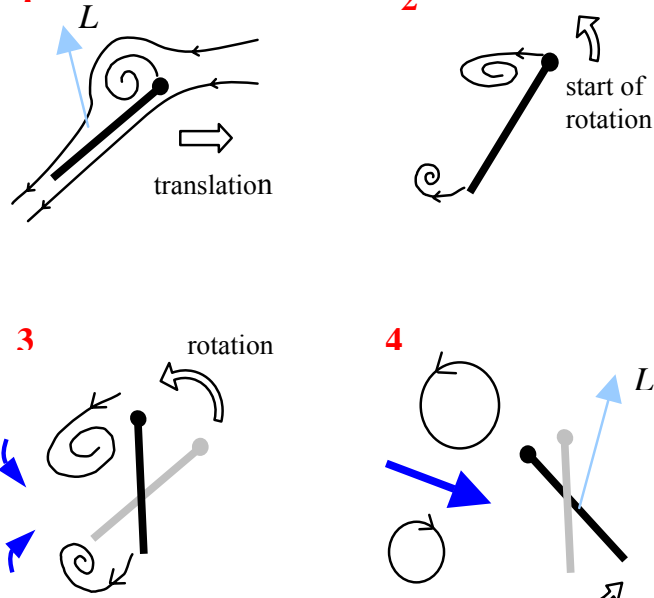

4

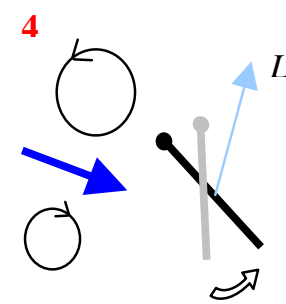

Figure 4. Wake capture process. Adapted from Dickinson, and Sane [11].

Leading Edge Vortex (LEV) that forms on the wing and provides a low pressure zone on the top the wing increasing the amount of lift that is generated. See Figure 3.

The generation of a LEV in a 2-D wing translation subsequently sheds itself. In a 3-D flapping wing, there is stability factor that helps to attenuate the growth of the LEV and allows it to stay attached to the wing throughout the entire downstroke providing a lift contribution of up to $2 / 3$ of the insects' body weight [14]. Ellington and Van Den Berg's smoke visualization study of model hawkmoth wings (Re 2000) concluded that the stability of LEV was the result of spanwise axial flow that feeds vorticity from the LEV (also observed by Maxworthy [15]) and thereby reduces its growth [14]. Birch and Dickinson's Digital Particle Image Velocimetry (DPIV) study of a scaled model fruit fly ( $\operatorname{Re} \sim 140)$ accounted for the stability of the LEV by the attenuation from the induced downwash effects [16]. The question of LEV stability remains an open item and it may be ultimately dependent on the Re flight regime that is studied.

\section{Wake Capture}

Wake Capture is the term used for wing-wake interactions in which there is a re-use of energy from previously shed vortices. Due to the cyclic pattern in the flapping wings, it is possible that the wing at certain points along its path will encounter the wake (disturbed flow) of a previous wing phase. For example: after the downstroke translation phase of the wing it will reverse direction at the supination phase where it undergoes a rapid rotation. During this rotation the wing may shed leading and trailing edge vortices that in turn creates an 'inter-vortex' stream that engages the wing before it begins its upstroke and adds to the resultant force vector on the wing; see step 4 in Figure 4 [11]. The above theoretical interpretation is corroborated with experimental results where peak wing forces seen at the point of stroke reversal were shown to be a consequence of wing-wake interactions by using force measurements and DPIV flow visualization [18,19]. This is in contrast to a numerical simulation performed by Sun and Tang, who concluded that the force peaks at stroke reversal were simply due to the acceleration of wing rotation and that there was no evidence of wing-wake interactions contributing to the resultant force [19].

\section{Rotational Mechanisms - Kramer Effect}

There is a characteristic peak force at the point of stroke reversal when the wing undergoes a rapid rotation due not only to possible wing-wake interactions but also to the Kramer effect [20]. The 
Kramer effect is the deviation from the Kutta condition whereby the rear stagnation point of the wing moves away from the trailing edge when a wing undergoes a simultaneous translation and rotation about spanwise axis [11]. The fluid generates additional circulation in order to try to re-establish the Kutta condition. Since the Kutta condition takes a finite amount of time to develop and if the wing continues to rotate during this

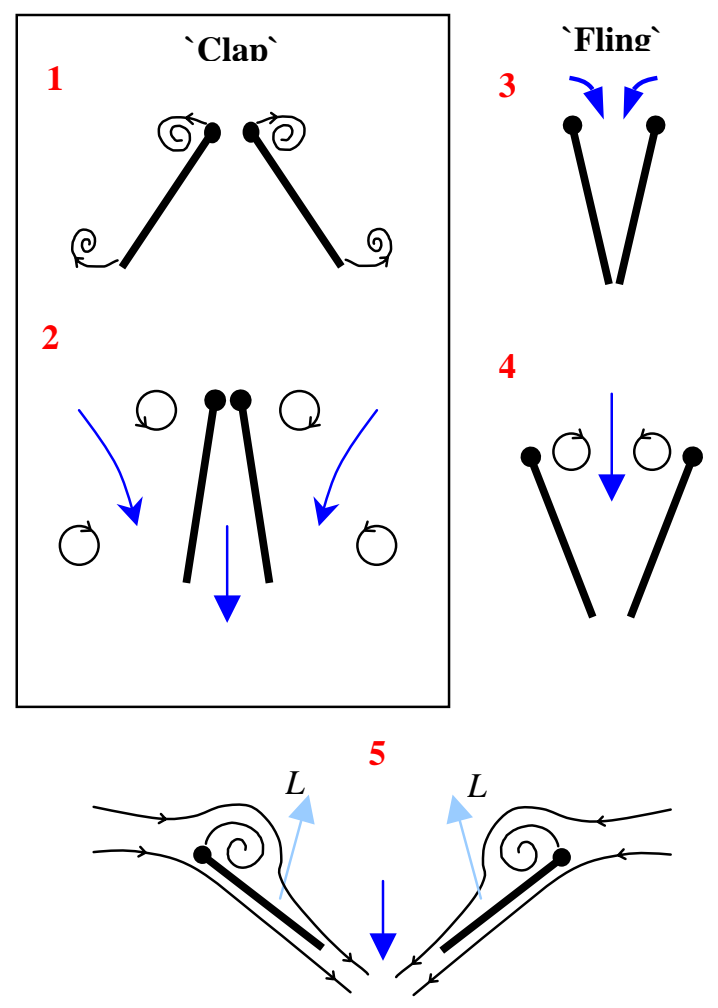

Figure 5. 'Clap \& Fling' schematic.

Adapted from Sane [11]. The circle on the end of the wing cross section (black line) represents the leading

time then the fluid continues to add circulation until the Kutta condition is reached. This added circulation, depending on the direction of rotation, can add to the resultant force vector and hence increase the 'overall lift'. The Kramer effect has also been termed 'rotational forces' by Sane and Dickinson [13]. Similar force peaks, as stated above, by Sun and Tang seem to corroborate the Kramer effect [19].

\section{Rotational Mechanisms - Clap \& Fling}

The 'clap \& fling' was one of the first unsteady aerodynamic mechanisms proposed by Weis-Fogh to explain the high lift characteristics of insect flight [21]. The 'clap \& fling' occurs on the dorsal side of the insect when the two wings first come up and make contact with each other; the 'clap'. See Figure 5. The two wings approach each other with their leading edges and as they 'clap' together they shed vortices that accumulated during the upstroke. This wake vorticity induces a downwash of air and coupled with the air between the wings being 'pushed' down contributes a vertical force and increase the 'overall lift'. The wings then 'fling' apart by rotating about their trailing edge and allowing air to rush into the low pressure zone between the wings. This oncoming air creates attached vortices as the wings translate away from each other. The movement of the wings induces a relative oncoming velocity vector and combined with the attached leading edge vortices the resultant force contributes to the 'overall lift'. The 'clap \& fling' can also be thought of as being two separated aerodynamic phenomena. It is used by insects for hovering and low speed flight as well as for birds to maximize lift during takeoff. Insects may also employ slight variations of this mechanism such as the 'clap \& peel' or the 'near clap \& fling' [8].

\section{Prototypes}

An earlier report prepared for the Canadian Space Agency (CSA) focused on the investigation of Martian flight vehicles [22]. The report identified flapping wing devices as a possible means of Mars flight and proposed the following prototypes in order to ascertain the feasibility of this flight mode. The major advantage of flapping wing flight is that it generates higher lift coefficients. This desirable characteristic must be weighed with the complexity associated with such a design, both in understanding the theory behind the design and the successful application of the technology in a more mature operational prototype. The purpose of these prototypes is to quantify the lift produced by each mechanism and therefore the parameters such as size, power, control etc. are reserved for later studies.

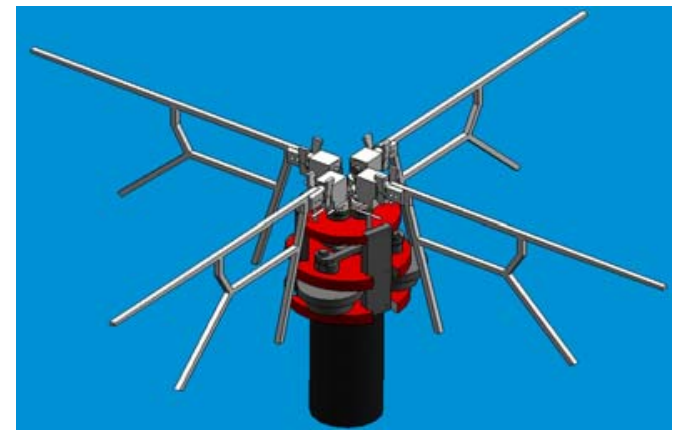

Figure 6. Four-wing flapping prototype

[22]. 


\section{Rotorcraft}

The Rotorcraft prototype is a simple model where a small motor turns a pair of wings in a rotary direction (i.e. helicopter). This rotary device is used in this study as an experimental basis due to its simplicity and better-known aerodynamics.

\section{Flapping Wing (4-wing design)}

This flapping prototype is similar to a flapping wing device that was designed and built at the University of Toronto Institute for Aerospace Studies (UTIAS). This prototype consists of four semi-controlled wings distributed symmetrically about a central axis of rotation pointing their wings tips in the radially outward direction with their leading edges facing vertically upward. See Figure 6. Each wing has a peak-to-peak amplitude of 85 degrees and one complete cycle of a wing movement consists of a 'clap' with both wings adjacent to itself. Essentially, this prototype is modeled after the 'clap \& fling' unsteady aerodynamic mechanism. A motor drives a spur gear transmission mechanism that in turn drives a set of three four-bar linkages that provide the oscillatory mechanism creating the flapping motion. Torsion springs are installed at the base of each individual wing subassembly in order to minimize the amount of torque required to drive the system. The entire mechanism has been designed to symmetrically distribute the mass about the center axis of rotation in order to avoid any operational imbalances. The wings have a passive angle of attack system whereby the inertia of the wing will cause the trailing edge of the wing to lag behind the leading edge, creating an angle of attack. Once two wings come close enough together the spring steel guides make contact and causes them to 'fling' apart and initiate a positive angle of attack in the opposite direction. This current prototype iteration has certain limitations that may inhibit the amount lift production. In insect flight, the wing amplitude can be twice that of the prototype. This extra travel time may result in the generation of stronger vorticity that could lead to greater downwash effects leading to the 'clap' and prolonged LEV attachment following the 'fling'. A recent numerical study by $\mathrm{Wu}$ and Sun demonstrated the importance of varying the wing amplitude and/or the stroke frequency with reference to force generation; the study calculated a $32 \%$ increase in mean lift force with only a $15 \%$ increase in stroke amplitude [23]. Small modifications of this design have been outlined or suggested in the previous report that could give the system an active angle of attack as well as an increased wing amplitude motion in later iterations.

\section{Rotary-Flapping Mechanism}

The mechanism of this prototype (as well as various adaptations) was invented by Canadian Clément Therriault and has a United States Patent No. US

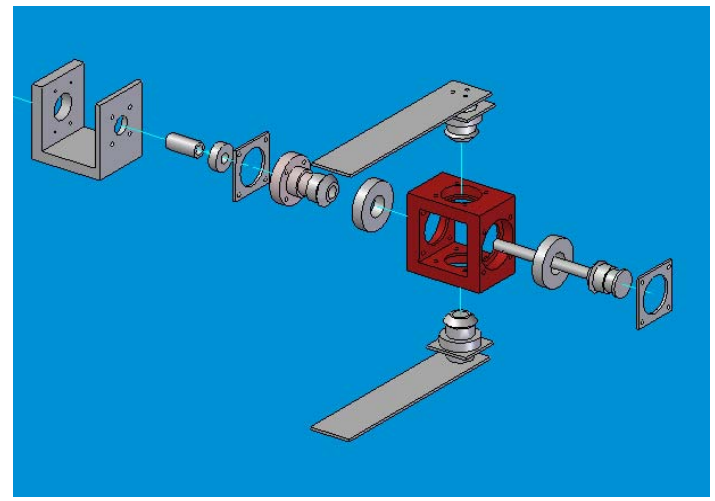

Figure 7. Exploded view of the rota-flap mechanism [22]. The motor is not shown.

6,227,483 B1, May 8, 2001. It is a simple and efficient hybrid system that incorporates both rotary and flapping-like motions (rota-flap). A motor drives an input shaft that extends into a rectangular housing through a bearing and connects to a collection of bevel gears (gear ratio of one) that rotates a pair of wings relative to the housing with an axis or rotation that is perpendicular to the input shaft. See Figure 7. At the same time the housing rotates in the opposite direction to that of the motor and shaft due to the law of conservation of angular momentum. The result is that the wing tips trace out a figure-eight-like pattern resembling the wing tip path of insect and bird flight. The mechanical advantage to this mechanism is that the rotational motions of the motor and shaft, the housing and the wings always turn in the same direction respectfully. This eliminates the hindrance of battling inertia as is customary in other flapping wing devices such as the earlier described 4-wing mechanism. The exact aerodynamic consequence of the above wing motion is yet to be determined. With the aid of force measurements and flow visualization we will be able to quantify as well as qualify the aerodynamic force generation. There are only two short sections of the wing path where the wings translate with an angle of attack that could produce an 'overall lift' force through a traditional conventional aerodynamic means. However the rotational movements of the wing relative to the ground could result in unsteady aerodynamic force production. Wake capture could also play an important role and 
the 'clap \& fling' could be exploited here if two sets of wings are operating adjacent to each other. As well, the Kramer effect might be of increased importance here due to the fact that the wings are constantly in a state of translation and rotation. Numerous adaptations of this mechanism are possible and will be used if deemed necessary.

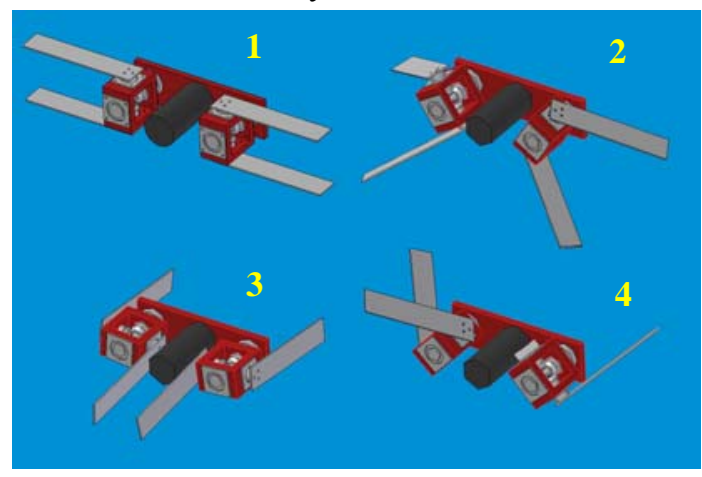

Figure 8. Wing motion on an adapted rota-flap mechanism [22]. The figure shows the sequence of two sets of wings operating adjacent to each other with a motor in between them.

\section{Wings}

Although the above prototypes are different with respect to the motions of the wings, their design necessitates the use of these wings and therefore the wing design parameter becomes important. The rotorcraft is currently using a set of standard model helicopter wings. The first test iteration of the other prototypes will use flat plates followed by wings of varying parameters in order to observe the resultant change of force and determine the effects of each parameter.

\section{Next Steps}

As of the submission of this paper there has been no lift data extracted yet. Preliminary experimental tests with the rotorcraft in a mini-domed controlledenvironment have been performed in order to validate the experimental procedure. This exercise resulted in the identification of various errors associated with the test bed. Most notably is the load cell sensitivity to vibrations that are inherent in the test bed design. These small vibrations can impact the results a great deal since the expected force magnitudes might not be substantially large.

The next steps involve the final integration of the flapping wing and rota-flap mechanisms as well as determining the most efficient method to measure the forces produced. The rotorcraft used a single axis micro-load cell which is sufficient for an aerodynamically well-known design where the expected forces would be in one direction; vertically upward. The aerodynamics of the other prototypes is unknown and more importantly, the direction of the 'overall lift' vector is unknown. It would be prudent to be able to measure the forces produced in a set of three mutually orthogonal axes. This could be either done with a multi-axis load cell, multiple load cells or strain gauges, using other force transducers, or varying the mounting orientation of the prototype in the different axes. The later technique might introduce some other experimental errors and would most likely introduce other complexities in the set ups.

The initial experimental validation tests used a minidomed vacuum chamber. The actual lift experiments will be conducted in the CSA's larger vacuum chamber. A range of atmospheric densities will be tested since the density of Mars' atmosphere changes depending on location and season. The chamber is also important for testing of candidate actuators that are currently in development.

The reality of the subsequent testing is that the abundance of parameters in flapping wing flight coupled with the time dependence for force production creates a complex system of variables and timing. Which must be carefully investigated and taken into account so not to hastily discount a mechanism without adequately 'tweaking' the parameters through a broad enough range in order to converge on a configuration that delivers the goal of high 'overall lift'.

Numerical solutions would provide some complementary results for comparison and would enable further analysis and understanding of the aerodynamics involved for the prototypes.

\section{Acknowledgment}

Gabriel Desmarais, Department of National Defence, CSA, NSERC.

\section{References}

[1] D. Anubhav, et al, "Design of a Martian Autonomous Rotary-Wing Vehicle" Journal of Aircraft, vol. 40, No. 3, 2003, pp. 461-472.

[2] R. Sullivan et al, "Results of the Imager for Mars Pathfinder windsock experiment" Journal of Geophysical Research, vol. 105, No. E10, 2002, pp. 24,547-24,562.

[3] L. A. Young, G.A. Briggs, "Design Opportunities and Challenges in the Development of Vertical Lift 
Planetary Aerial Vehicles" presented at the American Helicopter Society International Vertical Lift Aircraft Design Specialist's Meeting, San Francisco, CA, January 19-21, 2000.

[4] C.P. Ellington, "The Novel Aerodynamics of Insect Flight: Applications to Micro-Air vehicles" Journal of Experimental Biology, vol. 202, 1999, pp. 3439-3448.

[5] C.P. Ellington, "The Aerodynamics of Hovering Insect Flight. I. The Quasi-Steady Analysis" Phil. Trans. R. Soc. Lond. B, vol. 305, 1984, pp. 1-15.

[6] C.P. Ellington, "The Aerodynamics of Hovering Insect Flight. II. Morphological Parameters" Phil. Trans. R. Soc. Lond. B, vol. 305, 1984, pp. 17-40.

[7] C.P. Ellington, "The Aerodynamics of Hovering Insect Flight. III. Kinematics" Phil. Trans. R. Soc. Lond. B, vol. 305, 1984, pp. 41-78.

[8] C.P. Ellington, "The Aerodynamics of Hovering Insect Flight. IV. Aerodynamic Mechanisms" Phil. Trans. R. Soc. Lond. B, vol. 305, 1984, pp. 79-113.

[9] C.P. Ellington, "The Aerodynamics of Hovering Insect Flight. V. A Vortex Theory" Phil. Trans. R. Soc. Lond. B, vol. 305, 1984, pp. 115-144.

[10] C.P. Ellington, "The Aerodynamics of Hovering Insect Flight. VI. Lift and Power Requirements" Phil. Trans. R. Soc. Lond. B, vol. 305, 1984, pp. 145-181.

[11] S.P. Sane, "The aerodynamics of Insect Flight" J. Exp. Biol., vol. 206, 2003, pp. 4191-4208.

[12] Anderson, J.D., Fundamentals of Aerodynamics $2^{\text {nd }}$ edition, McGraw-Hill Inc., N.Y., USA, 1991.

[13] S.P. Sane, M.H. Dickinson, "The aerodynamic effects of wing rotation and a revised quasi-steady model of flapping flight" J. Exp. Biol. vol. 205, 2002, pp. 10871096.

[14] C. Van Den Berg, C.P. Ellington, "The threedimensional leading-edge vortex of a 'hovering' model hawkmoth" Phil. Trans. R. Soc. Lond. B, vol. 352, 1977, pp. 329-340.

[15] T. Maxworthy, "Experiments on the Weis-Fogh mechanism of lift generation by insects in hovering flight. Part 1. Dynamics of the "fling" J. Fluid. Mech., vol. 93, 1979, pp. 47-63.

[16] J.M. Birch, M.H. Dickinson, "Spanwise flow and the attachment of the leading-edge vortex on insect wings" Nature vol. 412, 2001, pp. 729-733.

[17] M.H. Dickinson, F. Lehmann, S.P. Sane, "Wing Rotation and the Aerodynamic Basis of Insect Flight" Science, vol. 284, 1999, pp. 1954-1960.

[18] J.M. Birch, M.H. Dickinson, "The influence of wingwake interactions on the production of aerodynamic forces in flapping flight" J. Exp. Biol. vol. 206, 2003, pp. 2257-2272.

[19] M. Sun, J. Tang, "Unsteady aerodynamic force generation by a model fruit fly wing in flapping motion" J. Exp. Biol. vol. 295, 2002, pp. 55-70.

[20] M. Kramer, "Die Zunahme des Maximalauftriebes von Tragflugeln bei plotzlicher Anstellwinkelvergrosserung (Boeneffekt). Z. Flugtech. Motorluftschiff. vol. 23, 1932, pp. 185-189.

[21] R. Weis-Fogh, "Quick Estimates of Flight Fitness in Hovering Animals, including Novel Mechanisms for
Lift Production" J. Exp. Bio., vol. 59, 1973, pp. 169230.

[22] G. Desmarais, "Martian Flight Study: prepared for the Canadian Space Agency" M.S. project, Concordia University, Montreal, Canada, 2003.

[23] J.H. Wu, M. Sun, "Unsteady aerodynamic forces of a flapping wing” J. Exp. Biol. vol. 207, 2004, pp.11371150. 